\title{
Transition of the hydration state of a surfactant accompanying structural transitions of self-assembled aggregates
}

\author{
M Hishida ${ }^{1} \ddagger$ and $\mathbf{K}$ Tanaka $^{1,2}$ \\ ${ }^{1}$ Institute for Integrated Cell-Material Sciences, Kyoto University, Kyoto 606-8501, \\ Japan \\ ${ }^{2}$ Core Research for Evolutional Science and Technology (CREST), Japan Science and \\ Technology Corporation (JST), Japan \\ E-mail: hishida@scphys.kyoto-u.ac.jp, kochan@icems.kyoto-u.ac.jp
}

\begin{abstract}
What role does water play in the self-assembly of soft materials? To understand the correlation between the hydration state of a nonionic surfactant and the self-assembly of soft materials to various structures, terahertz time-domain spectroscopy has been performed for a $\mathrm{C}_{12} \mathrm{E}_{5}$ solution with complementary use of small-angle X-ray scattering. Precise observations of the hydration state show clearly that transitions of the hydration state are accompanied by structural phase transitions of the surfactant from hexagonal to micelle to lamellae. These transitions of hydration state suggest that water is not a homogeneous solvent, and the interaction between water and the soft material is important for self-assembly.
\end{abstract}

\section{Introduction}

Water is the most basic solvent for biomolecules and industrial products. However, the role water plays in their functions is not yet fully understood. Lipids and surfactants, whose common characteristics include a hydrophilic head group and hydrophobic tail(s) in a single molecule, are good model systems for investigating the role of water, since they are often used in cells and in industrial products such as biomembranes, detergents, cosmetics, and foods. These amphiphilic molecules are known to exhibit structural transitions of aggregates - such as between micelle, bilayer, hexagonal and cubic structures - on the mesoscale. These mesoscopic structural transitions are keys to understanding biological functionality and producing industrial materials. The physicochemical mechanisms of these transitions are not yet fully understood, and it is still discussed just using the molecular morphologies of the lipid or surfactant in many cases $[1,2]$. However, these transitions should be based on the interactions among tails,

$\ddagger$ Present address: Division of Chemistry, Faculty of Pure and Applied Sciences, University of Tsukuba, 1-1-1 Tenno-dai, Tsukuba 305-8577, Japan. Present e-mail: hishida@chem.tsukuba.ac.jp 
head groups and water molecules. In the present study, we focus on the interactions between head groups and water, which is called hydration water.

The hydration water at the surface of a lipid or surfactant has been studied mainly by NMR or neutron scattering by focusing on the molecular dynamics of water itself with mixing rotational and translational dynamics [3-5]. With these methods, the observable time scales are from $10^{-9}$ to $10^{-11} \mathrm{~s}$. However, to understand the hydration state precisely, it is necessary that only a specific dynamics is focused. Further, the rotational relaxational time scale of water molecules lies in the range $10^{-13}-10^{-12} \mathrm{~s}[6-8]$. This implies that only strongly perturbed hydration water, called the first hydration shell, can be observed by these techniques. Thus, in many cases, only a single layer of water has been defined as the hydration layer [3]. However, it has been considered that much more perturbed hydration water exists beyond the first hydration shell, if slightly perturbed water molecules are included as the hydration water [1]. The amount of hydration water depends highly on its definition and the cutoff time scale of molecular dynamics.

To understand precisely the hydration state of a solute including slightly perturbed hydration water, it is necessary to observe the molecular dynamics of water on a time scale of $10^{-13}-10^{-12} \mathrm{~s}$. Since until recently no good experimental methods for making such observations have existed, molecular dynamics (MD) simulation is the only method available to investigate the fast water dynamics [9-13]. MD simulations have predicted the long-range hydration state up to 1-10 nm from the surface of the solute. Fortunately, rapid innovation of ultrafast laser technology in this decade has stimulated the progress of terahertz spectroscopy, and it is now becoming possible to observe the molecular dynamics on time scales of $10^{-13}-10^{-12} \mathrm{~s}$ using this technique $[7,11,14-17]$.

Thus, recently, to investigate experimentally the hydration effect of a lipid bilayer (a model biomembrane) on a molecular dynamics of water in the time scales of $10^{-13}-10^{-12}$ $\mathrm{s}$, terahertz time-domain spectroscopy (THz-TDS) was applied to a phospholipid solution with complementary use of small-angle X-ray scattering (SAXS) for obtaining structural information of lamellae. As a result, a correlation between the hydration state and the lamellar repeat distance of the lipid membranes is observed when the concentration of lipid is changed. This correlation verifies that the hydration of the lipid bilayer becomes long-range effect up to 4-5 water layers for a single leaflet of a bilayer, i.e. 8-10 water layers of hydration water exist between two lipid membranes, when we define the slightly perturbed water as the hydration water [18]. This length of the long-range hydration effect is about $1 \mathrm{~nm}$ from the surface, which is much longer than the results by NMR or neutron scattering which observe only strongly perturbed water as the hydration water, i.e., the first hydration shell [3-5]. Similar results of the long-range hydration effect have been reported in a water-in-oil emulsion of AOT surfactant [19].

This hydration effect has a comparable length scale to that of some interactions that dominate the self-assembly of soft materials, such as van der Waals or electrostatic interactions. It also has the same length scale as the characteristic self-assembled 
structures of lipid and surfactant aggregates (micelles, bilayers, etc.). This means that the hydration state may strongly correlate with these interactions and self-assemblies, which has been ignored so far. In the present study, we investigate such a correlation between hydration state and self-assembled structures of a nonionic surfactant by using terahertz time-domain spectroscopy (THz-TDS) and SAXS. As a result, a certain correlation was verified through lamellar-micelle-hexagonal transitions.

\section{Materials and methods}

We used the nonionic surfactant $\mathrm{C}_{12} \mathrm{E}_{5}$ (pentaethylene glycol monododecyl ether, purity $>98 \%$, Wako Pure Chemical Industries) as a sample without further purification. $\mathrm{C}_{12} \mathrm{E}_{5}$ is known to have complex phase transitions with respect to temperature and ratio to water (see Fig. 1(a)) [20]. $\mathrm{C}_{12} \mathrm{E}_{5}$ was mixed with pure water (MilliQ) to be $45 \mathrm{wt} \%$ $\mathrm{C}_{12} \mathrm{E}_{5}$ and $55 \mathrm{wt} \%$ water. The temperature was controlled between $10^{\circ} \mathrm{C}$ and $70^{\circ} \mathrm{C}$ in steps of $5^{\circ} \mathrm{C}$ for both THz-TDS and SAXS.

The THz-TDS measurement system was constructed by us as reported previously $[14,17,18,21]$. A terahertz wave was generated by shining a femtosecond pulsed laser (Femtolite, IMRA America, Inc.; $780 \mathrm{~nm}, 100 \mathrm{fs}, 50 \mathrm{MHz}$ ) onto a photoconductive switch with a dipole antenna, and the wave was detected by the electro-optic sampling method using a (1 110$)$-oriented ZnTe crystal. To measure the absorption and phase shift of the $\mathrm{THz}$ wave by a solution with high precision, we performed an attenuated total reflection (ATR) measurement by using a silicon dove prism for the THz-TDS to avoid the strong absorption of the $\mathrm{THz}$ wave by the water. The penetration depth of the evanescent field of the $\mathrm{THz}$ wave was about $20 \mu \mathrm{m}$. The calculated complex dielectric functions from the wave form have high reliability in the $0.5-2.6 \mathrm{THz}$ region.

The SAXS measurements were performed at BL15A, Photon Factory, High Energy Accelerator Research Organization, Japan. The X-ray wavelength was $1.5 \AA$ and the X-rays were detected by using a CCD (C7300, Hamamatsu). The sample-to-detector distance of about $1 \mathrm{~m}$ was calibrated using standard samples (lead stearate and silver behenate).

\section{Results and discussion}

Figure 1(a) shows the phase diagram of $\mathrm{C}_{12} \mathrm{E}_{5}$ with respect to the temperature and the mixing ratio with water [20]. For $45 \mathrm{wt} \% \mathrm{C}_{12} \mathrm{E}_{5}$ solution, the phase changes from a hexagonal $\mathrm{H}_{1}$ phase to a micellar $\mathrm{L}_{1}$ phase to a lamellar $\mathrm{L}_{\alpha}$ phase as temperature increases (see the arrow in Fig. 1(a)). We performed SAXS measurements to investigate the structural phase transitions and determined the transition temperatures as shown in Fig. 1(b). Between $10^{\circ} \mathrm{C}$ and $20^{\circ} \mathrm{C}$, the SAXS profiles indicate the hexagonal phase, where Bragg peaks appear at $q=q_{0}$ and $\sqrt{3} q_{0}$. The first Bragg peak is at about $q=q_{0}=0.12 \AA^{-1}$, which corresponds to the repeat distance of about $52 \AA$ between rod-like micellar structures in the hexagonal phase. From $25^{\circ} \mathrm{C}$ to $45^{\circ} \mathrm{C}$, the SAXS 

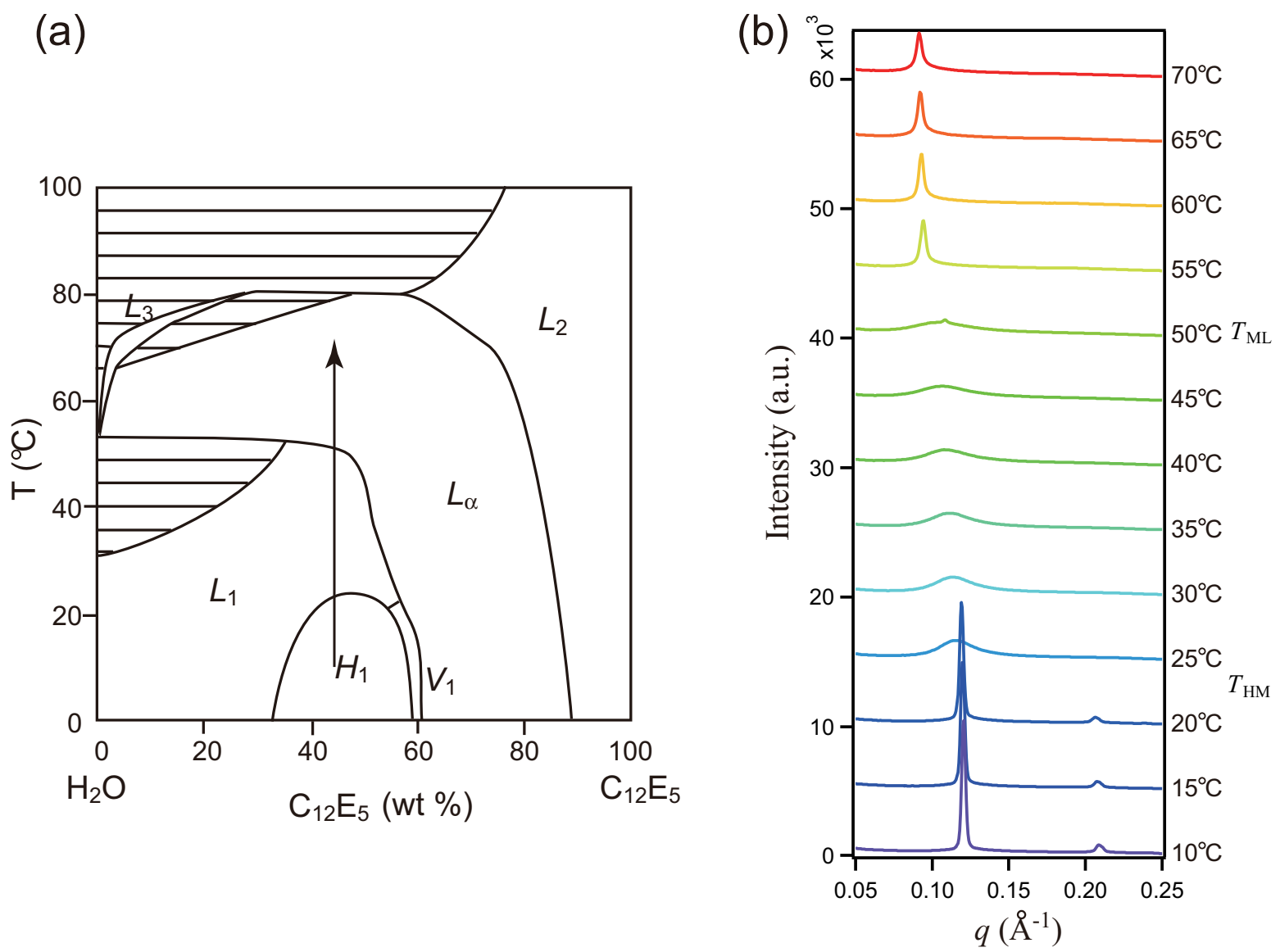

Figure 1. (a) The phase diagram of $\mathrm{C}_{12} \mathrm{E}_{5}$ from Ref. [20]. Our measurement region is shown by the arrow. (b) The SAXS profiles of $45 \mathrm{wt} \% \mathrm{C}_{12} \mathrm{E}_{5}$ solution at each temperature. $10^{\circ} \mathrm{C}-20^{\circ} \mathrm{C}$ : hexagonal $\mathrm{H}_{1}$ phase; $25^{\circ} \mathrm{C}-45^{\circ} \mathrm{C}$ : micellar $\mathrm{L}_{1}$ phase; $50^{\circ} \mathrm{C}$ : coexistence phase $\left(\mathrm{L}_{1}+\mathrm{L}_{\alpha}\right) ; 55^{\circ} \mathrm{C}-70^{\circ} \mathrm{C}$ : lamellar $\mathrm{L}_{\alpha}$ phase. $T_{H M}$ and $T_{M L}$ are the transition points.

profiles indicate the micellar phase, and the lamellar phase appears from $55^{\circ} \mathrm{C}$ to $70^{\circ} \mathrm{C}$, where the repeat distance calculated from the first Bragg peak is about $67 \AA$. At $50^{\circ} \mathrm{C}$, coexistence of micellar and lamellar phases is observed. Sometimes the coexisting phases are also observed at $45^{\circ} \mathrm{C}$. These results clearly show that the transition temperature from hexagonal to micellar phases $\left(T_{H M}\right)$ is about $23^{\circ} \mathrm{C}$, and that from micellar to lamellar phases $\left(T_{M L}\right)$ is about $50^{\circ} \mathrm{C}$.

For referencing the THz-TDS measurement of $45 \mathrm{wt} \% \mathrm{C}_{12} \mathrm{E}_{5}$ solution, we performed a THz-TDS measurement for pure water. Figure 2 shows the complex dielectric constants of pure water at each temperature in the $\mathrm{THz}$ frequency range. The imaginary part of the dielectric constant increases linearly with increasing temperature. In contrast, the real part of the dielectric constant increases below $0.7 \mathrm{THz}$ and decreases above $0.7 \mathrm{THz}$. These results are consistent with previously reported dielectric constants of pure water in the $\mathrm{THz}$ region [14]. The dielectric constant of water in this $\mathrm{THz}$ region is known to involve three contributions of dynamical modes: slow relaxation 
(Debye mode; relaxation time $\tau_{D} \approx 8$ ps at room temperature) caused by the rotational dynamics of the water molecule, fast relaxation $\left(\tau_{2} \approx 0.25 \mathrm{ps}\right.$ at room temperature), and an intermolecular stretching vibration mode (at about $5 \mathrm{THz}$ at room temperature) [14].

Since the variation of the imaginary part is larger and the errors in measurement were less for the imaginary part than for the real part, the value of the imaginary part of the dielectric constants of water at $0.5 \mathrm{THz}\left(\varepsilon_{w}^{\prime \prime}(0.5 \mathrm{THz})\right)$ are plotted against temperature as shown in Fig. 4(a) (open circles) to discuss the dielectric behavior of water qualitatively. $\varepsilon_{w}^{\prime \prime}(0.5 \mathrm{THz})$ is almost proportional to the temperature increase for pure water, where the coefficient of proportionality obtained is about $C=0.052$ from the fitting as $\varepsilon_{w}^{\prime \prime}(0.5 \mathrm{THz})=C \times T+D$ (where $T$ is temperature and $D$ is the intercept), which is shown as the solid line in Fig. 4(a) for the open circles. At $0.5 \mathrm{THz}$, the dielectric constant has contributions from the slow and fast relaxation modes. However, the fast relaxation mode is almost constant in this temperature range [14], and the slow component causes a change in the dielectric constant with changing temperature. Actually, the slow relaxation mode of water is well characterized by the imaginary part of the Debye function,

$$
\varepsilon_{D w}(\omega)=\frac{\Delta \varepsilon}{1+i \omega \tau_{D}}
$$

The calculated value of the imaginary part at $0.5 \mathrm{THz}, \varepsilon_{D w}^{\prime \prime}(0.5 \mathrm{THz})$, exhibits a completely linear behavior with temperature (with parameters calculated from Refs. $[22,23])$, and the proportionality coefficient is about $C=0.053$, which is almost the same as the measured coefficient. This indicates that the measured dielectric behavior of pure water as a function of temperature at around $0.5 \mathrm{THz}$ is caused by the change in the slow relaxational dynamics of water molecules. The contribution of the fast relaxational dynamics can be treated merely as background. The amplitude of the background is found to be about 0.95 for the entire temperature range.

Figure 3 shows the dielectric constants of $45 \mathrm{wt} \% \mathrm{C}_{12} \mathrm{E}_{5}$ solution in the $\mathrm{THz}$ region. In sharp contrast to the $\mathrm{THz}$ dielectric behavior of pure water, the dielectric constant of $\mathrm{C}_{12} \mathrm{E}_{5}$ solution does not exhibit a linear change with temperature. For example, for the imaginary part, a clear gap is observed between $20^{\circ} \mathrm{C}$ and $25^{\circ} \mathrm{C}$, and between $25^{\circ} \mathrm{C}$ and $70^{\circ} \mathrm{C}$, the dielectric constant increases nonlinearly. Further, for the real part, it increases over the whole frequency range with temperature, in contrast to the result of pure water.

The characteristic behavior is shown more clearly when the value of the imaginary part at $0.5 \mathrm{THz}$ is plotted as shown by the closed circles in Fig. 4(a). The value is normalized to compare with the result of pure water (i.e., the raw value of the imaginary part is divided by the volume fraction of water in the solution using the relation shown in Ref. [24]). The normalized value of the solution, $\varepsilon_{s}^{\prime \prime}(0.5 \mathrm{THz})$, shows transition-like behavior at about $23^{\circ} \mathrm{C}$ and $50^{\circ} \mathrm{C}$, which is consistent with the structural transition temperatures $T_{H M}$ and $T_{M L}$ observed in SAXS. At $T_{H M}, \varepsilon_{s}^{\prime \prime}(0.5 \mathrm{THz})$ shows a discrete jump and a small change of the proportionality as a function of temperature $(C=0.039$ to 0.032 ). At $T_{M L}$, only the proportionality shows a large change from 0.032 to 0.010 . 


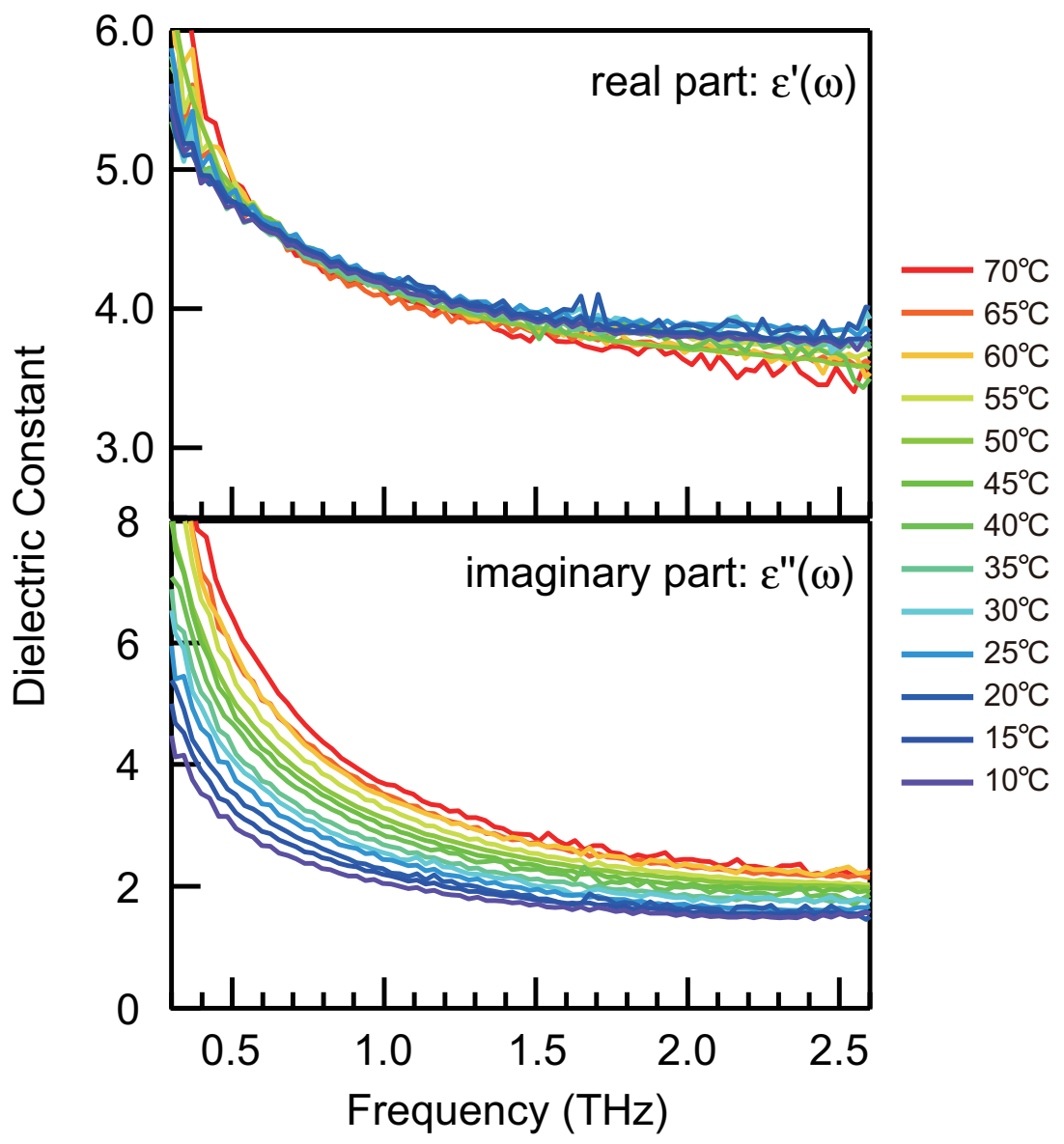

Figure 2. The dielectric constants of pure water in the terahertz region at each temperature. The upper figure is the real part and the lower figure is the imaginary part of the dielectric constant.

Further, we performed a THz-TDS measurement for pure $\mathrm{C}_{12} \mathrm{E}_{5}$ at room temperature (micellar $\mathrm{L}_{1}$ phase) and observed that the pure $\mathrm{C}_{12} \mathrm{E}_{5}$ exhibits no absorption of light in the $\mathrm{THz}$ frequency region. Therefore, the THz-TDS spectra for $45 \mathrm{wt} \% \mathrm{C}_{12} \mathrm{E}_{5}$ solution reflect only the dynamics of water molecules in the solution. Thus, these THzTDS and SAXS results strongly indicate that the hydration state of the surfactant undergoes a transition accompanying the structural transition between hexagonal, micellar, and lamellar phases. It has been suggested that when water molecules become the hydration water, only the slow relaxational mode is influenced and the fast relaxational mode remains unchanged $[17,18]$. The slow relaxational mode of the hydration water becomes much slower than that of bulk water, and it is not observed in the $\mathrm{THz}$ frequency range.

Here, we briefly summarize our present results and previous reports: (1) The fast relaxation component of the dielectric behavior of water is constant and the slow component only changes with temperature. (2) Only the slow relaxational component is influenced by the hydration effect. (3) Pure $\mathrm{C}_{12} \mathrm{E}_{5}$ does not absorb $\mathrm{THz}$ waves. (4) The 


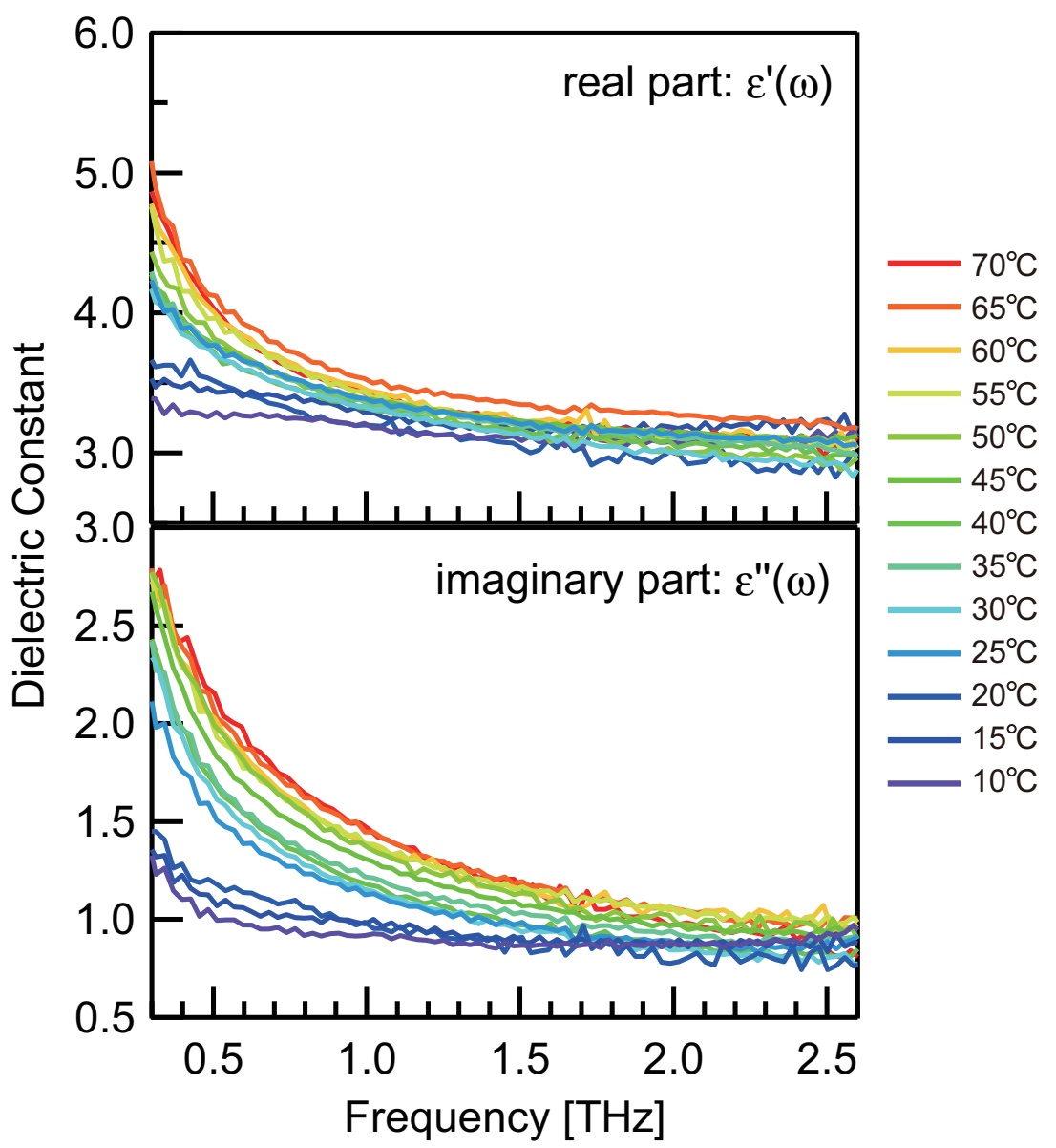

Figure 3. The dielectric constants of $45 \mathrm{wt} \% \mathrm{C}_{12} \mathrm{E}_{5}$ solution in the terahertz region at each temperature. The upper figure is the real part and the lower figure is the imaginary part of the dielectric constant.

$\mathrm{THz}$ spectra of $\mathrm{C}_{12} \mathrm{E}_{5}$ solution exhibit a transition-like behavior. These facts indicate that the characteristic behavior of $\varepsilon_{s}^{\prime \prime}(0.5 \mathrm{THz})$ of $\mathrm{C}_{12} \mathrm{E}_{5}$ solution is caused by the change of the slow relaxational mode of water by the hydration effect of the surfactant. Since the intensity of the slow relaxational mode is proportional to the amount of bulk water in the solution, we can roughly estimate how much water becomes hydration water by the reduction of $\varepsilon_{s}^{\prime \prime}(0.5 \mathrm{THz})$ of the $\mathrm{C}_{12} \mathrm{E}_{5}$ solution from $\varepsilon_{w}^{\prime \prime}(0.5 \mathrm{THz})$ of pure water. The hydration number per surfactant molecule is calculated as

$$
\begin{aligned}
n_{h} & =N \frac{\varepsilon_{D w}^{\prime \prime}(0.5 \mathrm{THz})-\varepsilon_{D s}^{\prime \prime}(0.5 \mathrm{THz})}{\varepsilon_{D w}^{\prime \prime}(0.5 \mathrm{THz})} \\
& =N \frac{\varepsilon_{w}^{\prime \prime}(0.5 \mathrm{THz})-\varepsilon_{s}^{\prime \prime}(0.5 \mathrm{THz})}{\varepsilon_{w}^{\prime \prime}(0.5 \mathrm{THz})-\varepsilon_{B}^{\prime \prime}},
\end{aligned}
$$

where $N$ is the total number of water molecules per surfactant $\left(=27.6\right.$ for $45 \mathrm{wt} \% \mathrm{C}_{12} \mathrm{E}_{5}$ solution $), \varepsilon_{D s}^{\prime \prime}(0.5 \mathrm{THz})$ is the imaginary part of the slow relaxational component of the surfactant solution at $0.5 \mathrm{THz}$, and $\varepsilon_{B}^{\prime \prime}$ is the background at $0.5 \mathrm{THz}$ mainly resulting from the fast relaxational mode, which is known to be about 0.95 from the result of 
(a)

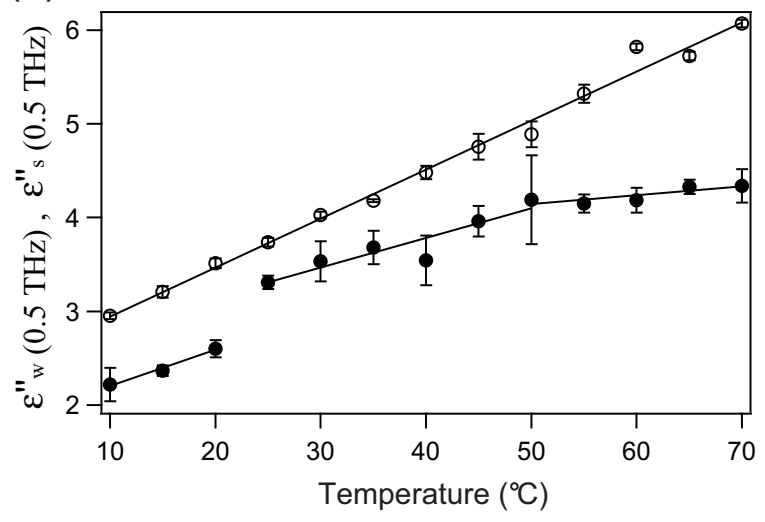

(b)

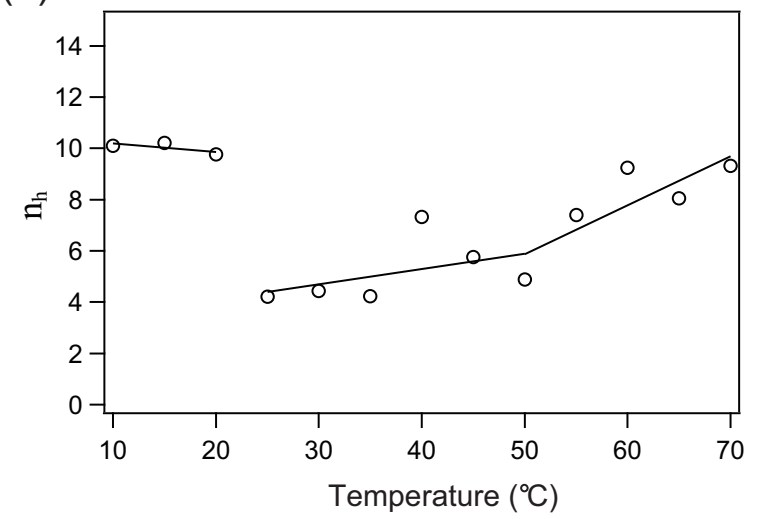

Figure 4. (a) The value of the imaginary part of the dielectric constant at $0.5 \mathrm{THz}$ with respect to temperature. Open circles are for pure water and closed circles are for the normalized value for $45 \mathrm{wt} \% \mathrm{C}_{12} \mathrm{E}_{5}$ solution. The solid lines are the linear fitting result $\left(\varepsilon_{w, s}^{\prime \prime}(0.5 \mathrm{THz})=C \times T+D\right)$. For pure water $C=0.052$, whereas for $\mathrm{C}_{12} \mathrm{E}_{5}$ solution $C=0.039$ at $10^{\circ} \mathrm{C}-20^{\circ} \mathrm{C}, C=0.032$ at $25^{\circ} \mathrm{C}-45^{\circ} \mathrm{C}$, and $C=0.010$ at $50^{\circ} \mathrm{C}-$ $70^{\circ} \mathrm{C}$. (b) The hydration number per surfactant molecule at each temperature. The solid lines are the linear fits for each phase.

pure water.

In Fig. 4(b), the calculated hydration number $n_{h}$ is shown as a function of temperature. The hydration number at the hexagonal $\mathrm{H}_{1}$ phase is higher compared to other phases, and it is a constant of about 10 between $10^{\circ} \mathrm{C}$ and $20^{\circ} \mathrm{C}$. At $T_{H M}$, the hydration number exhibits a first-order transition-like behavior with a discrete jump from 10 to about 4 . It increases slightly for the micellar $L_{1}$ phase. The result for the micellar phase $\mathrm{L}_{1}\left(n_{h}=4-6\right)$ is consistent with previous THz-TDS results, where about 0.9 water molecules are calculated as the hydration water for a single ethylene glycol group $\left(\mathrm{C}_{12} \mathrm{E}_{5}\right.$ has five ethylene glycol groups) [25]. At $T_{M L}$, only the slope of the increase of the hydration number changes, like a second-order transition. The slope of the increase in the hydration number is steeper for the lamellar $\mathrm{L}_{\alpha}$ phase than for the micellar phase, reaching about 9.5 at $70^{\circ} \mathrm{C}$.

These results strongly suggest that the hydration state of a surfactant plays an important role in the self-assembly mechanism to various aggregates, which may become a general concept for various soft materials and biomolecules. Especially, the clouding phenomenon of nonionic polymers and nonionic surfactants has been considered to be caused by the transition of the hydration state [26]. The transition of the hydration state at the clouding point has been reported in the case of the nonionic polymer P(NIPAm) by a dielectric measurement in the gigahertz region [27]. The dielectric measurement of water in the $\mathrm{GHz}-\mathrm{THz}$ region seems to be a very powerful technique for understanding the hydration correlation to self-assembly. Our results indicate that the hydration state is important for the self-assembly of soft materials not only for the clouding phenomenon.

At present, the mechanism of the correlation between the hydration state and the 
self-assembled structure is still unclear. It is possible that the surface morphology of the surfactant aggregates in molecular scale such as curvature or molecular packing is the key factor of the correlation between the hydration state and the self-assemble structures. Our results of hydration number per a surfactant $\left(n_{h}=4-10\right)$ corresponds to one or two layers of water at the surfaces, which is almost in direct contact with the surfactant molecule. Such the hydration layer seems to be perturbed only by a nearby surfactant, and thus, the hydration number is determined only by the surface property of the aggregate in molecular scale. This length scale of the hydration layer is similar to that of cross-sectional area of a surfactant in these aggregates, which is determined by the curvature or molecular packing of surfactant in the aggregates.

\section{Conclusion}

In the present study, we performed $\mathrm{THz}$ time-domain spectroscopy to investigate the precise hydration state of the nonionic surfactant $\mathrm{C}_{12} \mathrm{E}_{5}$. By complementary use of small-angle X-ray scattering to determine the structural transitions of the surfactant, the hydration state of the surfactant is found to exhibit transitions accompanying the structural transitions of self-assembled structures from hexagonal to micellar to lamellar phases. The transitions of hydration states presented here imply that the behavior of water plays an important role in the self-assembly of soft materials. Consequently, water should not be treated as a homogeneous solvent for soft materials and biomolecules.

\section{Acknowledgments}

M.H. is supported by the Murata Science Foundation (H23-54) and by the iCeMS Cross-Disciplinary Research Promotion Project. The SAXS measurements were performed under the approval of the Photon Factory Program Advisory Committee (Nos. 2009G602 and 2009G217).

\section{References}

[1] Israelachvili J, 2010, Intermolecular and Surface Forces (London: Academic Press, Elsevier)

[2] Israelachvili J, Mitchell D J, Ninham B W J, 1976, Chem. Soc. Faraday Trans. 721525

[3] Milhaud J, 2004, Biochim. Biophys. Acta 166319

[4] König S, Sackmann E, Richter D, Zorn R, Carlie C, Bayerl T M, 1994, J. Chem. Phys. 1003307

[5] Swenson J, Kargl F, Berntsen P, Svanberg C, 2008, J. Chem. Phys. 129045101

[6] Buchner R, Barthel J, Stauber J, 1999, Chem. Phys. Lett. 30657

[7] Rønne C, Åstrand P O, Keiding S R, 1999, Phys. Rev. Lett. 822888

[8] Ratajska-Gadomska B, Bialkowski B, Gadomski W, Radzewicz Cz, 2006, Chem. Phys. Lett. 429 575

[9] Marrink S, Berkowitz M, Berendsen H J C, 1993, Langmuir 93122

[10] Zhang Z, Berkowitz M L, 2009, J. Phys. Chem. B 1137676

[11] Ebbinghaus S, Kim S J, Heyden M, Yu X, Heugen U, Gruebele M, Leitner D M, Havenith M, 2007, Proc. Natl. Acad. Sci. U.S.A. 10420749

[12] Makarov V A, Feig M, Andrews B K, Pettitt B M, 1998, Biophys. J. 75150 
[13] Ahmad M, Gu W, Geyer T, Helms T, 2011, Nat. Commun 2261

[14] Yada H, Nagai M, Tanaka K, 2008, Chem. Phys. Lett. 464166

[15] Tielrooij K J, Garcia-Araez N, Bonn M, Bakker H J, 2010, Science 3281006

[16] Heugen U, Schwaab G, Bründermann E, Heyden M, Yu X, Leitner D M, Havenith M, 2006, Proc. Natl. Acad. Sci. U.S.A. 10312301

[17] Arikawa T, Nagai M, Tanaka K, 2008, Chem. Phys. Lett. 45712

[18] Hishida M, Tanaka K, 2011, Phys. Rev. Lett. 106158102

[19] Mittleman D M, Nuss M C, Colvin V L, 1997, Chem. Phys. Lett. 275332

[20] Strey R, Schomäcker, Roux D, Nallet F, Olsson U, 1990, J. Chem. Soc. Faraday Trans. 862253

[21] Nagai M, Yada H, Arikawa T, Tanaka K, 2006, Int. J. IRMMW 27505

[22] Yastremskii P S, 1988, J. Structural Chem. 29483

[23] Vidulich G A, Evans D F, Kay R L, 1985, J. Phys. Chem. 71656

[24] Schrödle S, Hefter G, Kunz W, Buchner R, 2006, Langmuir 22924

[25] Arikawa T, Nagai M, Tanaka K, 2009, Chem. Phys. Lett. 47795

[26] Rosen M J, 1989, Surfactants and Interfacial Phenomena (New York: Wiley)

[27] Ono Y, Shikata T, 2008, J. Am. Chem. Soc. 12810030 